\title{
Correction to: Deep-sea glass sponges (Hexactinellida) from polymetallic nodule fields in the Clarion-Clipperton Fracture Zone (CCFZ), northeastern Pacific: Part I - Amphidiscophora
}

\author{
Daniel Kersken $^{1,2}$ • Dorte Janussen ${ }^{2} \cdot$ Pedro Martínez Arbizu ${ }^{1}$
}

Published online: 4 November 2017

(C) Senckenberg Gesellschaft für Naturforschung and Springer-Verlag GmbH Germany 2017

\section{Correction to: Mar Biodiv}

https://doi.org/10.1007/s12526-017-0727-y

In the originally published article, spicule size measurements in the descriptions of Tables 7, 9, 11, 15, 17 and 19 are indicated in centimeters $[\mathrm{cm}]$, which should be changed to micrometres $[\mu \mathrm{m}]$. Corrected tables are provided below.

The online version of the original article can be found at https://oi.org/ 10.1007/s12526-017-0727-y.

\footnotetext{
Daniel Kersken

daniel.kersken@senckenberg.de
}

1 German Centre for Marine Biodiversity Research (DZMB),

Senckenberg am Meer, Südstrand 44,

D-26382 Wilhelmshaven, Germany

2 Marine Zoology, Senckenberg Research Institute and Nature Museum, Senckenberganlage 25, D-60325 Frankfurt am, Main, Germany 
Table 7 Spicule size of Hyalonema (Cyliconemaoida) campanula, values in $[\mu \mathrm{m}]$ are given as follows: minimum - mean - maximum (number of spicules measured)

SMF 12098

Lendenfeld, 1915

Diactins

Length

$1175-\underline{1613}-3575(17) \quad 1000-1500$

Pinular diactins

Length

Chaonosomal pentactins

Length

Basis diameter

Choanosomal hexactins

Diameter

Macramphidiscs

Length

Umbel length

Umbel width

Mesamphidiscs

Length

Umbel length

Umbel width

Micramphidiscs

Length

Umbel length

Umbel width

Microhexactins

Diameter

Gastral pinular pentactins

$$
\begin{aligned}
& \text { Length } \\
& \text { Basis diameter }
\end{aligned}
$$

Dermal pinular pentactins

Length

Basis diameter

$-$

$730-1200$

$-\quad \leq 1000$

\begin{tabular}{l}
$-\quad 230-1000$ \\
\hline
\end{tabular}

$\begin{array}{lr}- & 550-1300\end{array}$

$40-49-55(30) \quad 48-84$

$35-\underline{45}-55(30) \quad 25-53$

$68-77-85(3) \quad 77-118$

$30-\underline{30}-30(3) \quad 30-43$

$28-\underline{32}-38(3) \quad 40-47$

$20-\underline{29}-40(30) \quad 18-26$

$5-\underline{9}-13(30) \quad 5-11$

$5-8-13(30) \quad 5.5-10$

$105-\underline{105}-105(1) \quad 50-100$

$810-\underline{939}-1000$ (14) $\quad 230-810$

$140-\underline{184}-220(11) \quad 86-160$

$100-165$

$74-116$ 
Table 9 Spicule size of Hyalonema (Cyliconemaoida) ovuliferum, values in $[\mu \mathrm{m}]$ are given as follows: minimum - mean - maximum (number of spicules measured)

\begin{tabular}{|c|c|c|c|c|c|c|}
\hline & SMF 12074 & SMF 12076 & SMF 12089 & SMF 11708 & SMF 11692 & $\begin{array}{l}\text { Schulze, } \\
1889\end{array}$ \\
\hline \multicolumn{7}{|l|}{ Diactins } \\
\hline Length & $660-\underline{1150}-2425(30)$ & $470-\underline{993}-2075(30)$ & $550-\underline{1016}-2300(30)$ & $775-\underline{1397}-2000(30)$ & $640-\underline{1005}-1600(30)$ & - \\
\hline \multicolumn{7}{|c|}{ 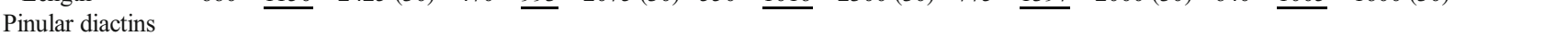 } \\
\hline Length & - & - & $980-\underline{1070}-1150(6)$ & $1150-\underline{1326}-1500(2)$ & $880-\underline{1694}-2425(30)$ & - \\
\hline \multicolumn{7}{|l|}{$\begin{array}{c}\text { Choanosomal } \\
\text { pentactins }\end{array}$} \\
\hline Length & $330-466-600(7)$ & $180-437-900(30)$ & $240-454-1000(9)$ & $450-642-925(6)$ & $240-446-575(13)$ & - \\
\hline Diameter & $410-\overline{639}-875(7)$ & $270-\overline{483}-700(30)$ & $360-\overline{537}-1225$ & $350-\overline{471}-650(6)$ & $340-\overline{713}-1200(13)$ & - \\
\hline \multicolumn{7}{|l|}{$\begin{array}{l}\text { Choanosomal } \\
\text { hexactins }\end{array}$} \\
\hline Diameter & $360-\underline{609}-1225(30)$ & $290-\underline{493}-760(30)$ & $290-493-930(30)$ & $500-\underline{947}-1550(24)$ & $310-746-1150(17)$ & - \\
\hline \multicolumn{7}{|c|}{ 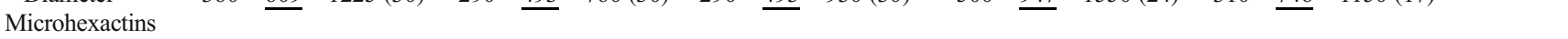 } \\
\hline Diameter & $50-\underline{73}-153(30)$ & $55-\underline{72}-108(30)$ & $63-\underline{78}-118(30)$ & - & - & $60-80$ \\
\hline \multicolumn{7}{|l|}{ Macramphidiscs } \\
\hline Length & $48-71-118(30)$ & $50-81-118(30)$ & $63-87-138(30)$ & $78-92-125(30)$ & $70-101-115(30)$ & $\leq 280$ \\
\hline Umbel length & $20-\overline{28}-40(30)$ & $13-\overline{28}-40(30)$ & $20-\overline{31}-48(30)$ & $33-\overline{39}-50(30)$ & $25-\underline{36}-43(30)$ & - \\
\hline Umbel width & $20-\underline{28}-43(30)$ & $13-\overline{27}-43(30)$ & $20-\underline{29}-48(30)$ & $35-\underline{41}-50(30)$ & $28-\underline{40}-48(30)$ & - \\
\hline \multicolumn{7}{|l|}{ Micramphidiscs } \\
\hline Length & $23-34-43(30)$ & $23-34-60(30)$ & $23-32-50(30)$ & $45-56-63(6)$ & $20-51-70(30)$ & $\geq 16$ \\
\hline Umbel length & $5-1 \overline{2}-18(30)$ & $5-1 \overline{0}-28(30)$ & $5-9-18(30)$ & $15-\overline{21}-25(6)$ & $8-1 \overline{8}-25(30)$ & - \\
\hline Umbel width & $78-\overline{12}-18(30)$ & $8-\overline{10}-18(30)$ & $8-\overline{9}-15(30)$ & $15-\overline{19}-23(6)$ & $10-\overline{1} 8-28(30)$ & - \\
\hline \multicolumn{7}{|l|}{$\begin{array}{l}\text { Gastral pinular } \\
\text { pentactins }\end{array}$} \\
\hline Length & $223-448-820(23)$ & $245-491-660(30)$ & $300-508-790(30)$ & - & $390-806-1375(9)$ & - \\
\hline Basis diameter & $75-1 \overline{03}-130(23)$ & $70-1 \overline{01}-143(30)$ & $75-105-140(30)$ & - & $100-\underline{166}-220$ & - \\
\hline \multicolumn{7}{|c|}{$\begin{array}{l}\text { Dermal pinular } \\
\text { pentactins }\end{array}$} \\
\hline Length & - & - & $150-\underline{201}-250(30)$ & - & $200-\underline{250}-360(20)$ & $300-400$ \\
\hline Basis diameter & - & - & $68-9 \overline{2-} 115(30)$ & - & $90-1 \overline{21}-163(20)$ & $80-100$ \\
\hline
\end{tabular}


Table 11 Spicule size of Hyalonema (Onconema) agassizi, values in $[\mu \mathrm{m}]$ are given as follows: minimum - mean - maximum (number of spicules measured)

\begin{tabular}{|c|c|c|}
\hline & SMF 12069 & Lendenfeld, 1915 \\
\hline \multicolumn{3}{|l|}{ Diactins } \\
\hline Length & $775-\underline{1210}-1775$ & $300-7000$ \\
\hline \multicolumn{3}{|l|}{ Uncinates I } \\
\hline Length & $310-\underline{427}-580(30)$ & $330-800$ \\
\hline \multicolumn{3}{|l|}{ Uncinates II } \\
\hline Length & $620-\underline{946}-1350(30)$ & - \\
\hline \multicolumn{3}{|l|}{ Acanthophores } \\
\hline Diameter & $85-\underline{94}-102(6)$ & $85-230$ \\
\hline \multicolumn{3}{|c|}{ Choanosomal pentactins } \\
\hline Length & $570-\underline{896}-1375$ & $300-1500$ \\
\hline Basis diameter & $330-\underline{707}-1800(30)$ & $500-2600$ \\
\hline \multicolumn{3}{|c|}{ Choanosomal hexactins } \\
\hline Diameter & $300-\underline{577}-950(30)$ & $400-6000$ \\
\hline \multicolumn{3}{|l|}{ Macramphidiscs } \\
\hline Length & $115-\underline{221}-290(30)$ & $48-310$ \\
\hline Umbel length & $43-\underline{65}-80(30)$ & $10-80$ \\
\hline Umbel width & $40-\underline{67}-75(30)$ & $10-86$ \\
\hline \multicolumn{3}{|l|}{ Mesamphidiscs } \\
\hline Length & $30-\underline{49}-60(30)$ & $35-64$ \\
\hline Umbel length & $10-\underline{18}-33(30)$ & $14-23$ \\
\hline Umbel width & $8-\overline{11}-15(30)$ & $8-14$ \\
\hline \multicolumn{3}{|l|}{ Micramphidiscs } \\
\hline Length & $15-\underline{21}-25(30)$ & $13-36$ \\
\hline Umbel length & $5-\underline{6}-8(30)$ & $4-13$ \\
\hline Umbel width & $5-\underline{6}-8(30)$ & $4.7-9$ \\
\hline \multicolumn{3}{|l|}{ Microhexactins } \\
\hline Diameter & $65-\underline{83}-118(30)$ & $44-160$ \\
\hline \multicolumn{3}{|c|}{ Gastral pinular pentactins } \\
\hline Length & $105-\underline{119}-130(30)$ & $83-153$ \\
\hline Basis diameter & $40-\underline{53}-68(30)$ & $40-170$ \\
\hline \multicolumn{3}{|c|}{ Dermal pinular pentactins } \\
\hline Length & $80-\underline{111}-123(30)$ & $82-114$ \\
\hline Basis diameter & $40-\underline{57}-80(30)$ & $42-76$ \\
\hline
\end{tabular}


Table 15 Spicule size of Hyalonema (Onconema) obtusum, values in [ $\mu \mathrm{m}]$ are given as follows: minimum - mean - maximum (number of spicules measured)

SMF 12053

Diactins

Length

Uncinates

Length

Pentactins

Length

Basis diameter

Hexactins

Diameter

Macramphidiscs

Length

Umbel length

Umbel width

Mesamphidiscs

Length

Umbel length

Umbel width

Micramphidiscs

Length

Umbel length

Umbel width

Microhexactins

Diameter

Gastral pinular pentactins

Length

Basis diameter

Dermal pinular pentactins

Length

Basis diameter
SMF 12054

SMF 12072

$1000-\underline{1733}-2250(30)$

$1000-\underline{1638}-2450(30)$

$500-3500$

$870-\underline{1608}-2425(30)$

$290-\underline{441}-570(30)$

$400-\underline{523}-690(30)$

$530-\underline{685}-840(2)$

$435-\underline{503}-570(2)$

$365-\underline{745}-1475(30)$

$320-\underline{345}-370(2)$

$100-\underline{103}-105$ (2)

$110-\underline{115}-120(2)$

$80-\underline{103}-130(13)$

$110-\underline{152}-180(7)$

$90-\underline{154}-220(15)$

$40-\underline{56}-70$ (15)

$35-\underline{55}-75$ (15)

$15-\underline{19}-45$ (30)

$5-\underline{6}-15(30)$

$5-\underline{6}-10(30)$

$45-\underline{63}-78(30)$

$45-\underline{61}-78(30)$

$90-127-160(30)$

$80-101-130$ (7)

$85-\underline{116}-150$ (7)

$65-\underline{127}-170(30)$

$120-\underline{154}-185$ (17)

$50-68-90(17)$
$130-145-160(9)$

$40-\underline{62}-70$ (9) $325-\underline{637}-950(30)$

$580-1100$

$525-1083-1475$ (3)

$470-1860$

$570-\underline{861}-1050$ (3)

$480-1500$

$390-\underline{857}-2025(30)$

$350-2000$

$135-\underline{262}-380(30)$

$235-356$

$55-\underline{89}-115(30)$

$70-100$

50 - $94-125$ (30)

$70-110$

$80-\underline{107}-140(30)$

$86-212$

$30-\underline{43}-60(30)$

$32-80$

$20-\underline{35}-50(30)$

$16-69$

$15-\underline{21}-38(30)$

$12-68$

$5-8-15(30)$

$5-\overline{7}-10(30)$

$3.8-25$

$4-26$

$33-\underline{73}-132(30)$

$42-80$

$73-145$

$70-180$

$-$

$155-\underline{195}-230(30)$

$137-172$

$50-66-80(30)$

$20-100$ 
Table 17 Spicule size of Hyalonema (Prionema) breviradix sp. nov., values in $[\mu \mathrm{m}]$ are given as follows: minimum - mean - maximum (number of spicules measured)

\begin{tabular}{ll}
\hline & SMF 12070 \\
\hline Diactins & \\
Length & $850-\underline{1464}-2475(30)$ \\
Pentactins & $375-\underline{1701}-2325(26)$ \\
Length & $430-\underline{1000}-2175(26)$ \\
Basis diameter & \\
Hexactins & $425-\underline{989}-2225(30)$ \\
Diameter & \\
Macramphidiscs & $390-\underline{458}-520(21)$ \\
Length & $120-\underline{135}-155(21)$ \\
Umbel length & $90-\underline{106}-130(21)$ \\
Umbel width & $130-\underline{214}-255(20)$ \\
Mesamphidiscs & $60-\underline{85}-105(20)$ \\
Length & $40-\underline{51}-60(20)$ \\
Umbel length & \\
Umbel width & $18-\underline{21}-25(30)$ \\
Micramphidiscs & $5-\underline{7}-10(30)$ \\
Length & $5-\underline{7}-8(30)$ \\
Umbel length & $65-\underline{122}-150(24)$ \\
Umbel width & \\
Microhexactins & $63-\underline{85}-108(30)$ \\
Diameter & $90-\underline{\underline{49}}-65(30)$ \\
Gastral pinular pentactins & \\
Length & \\
Basis diameter & \\
Dermal pinular pentactins & \\
Length & \\
Basis diameter & \\
Pinular hexactins & \\
Length & \\
Basis diameter & \\
\hline
\end{tabular}

Table 19 Spicule size of Poliopogon microuncinata sp. nov., values in $[\mu \mathrm{m}]$ are given as follows: minimum - mean - maximum (number of spicules measured)

\begin{tabular}{|c|c|}
\hline & SMF 11698 \\
\hline \multicolumn{2}{|l|}{ Diactins } \\
\hline Length & $17,262(1)$ \\
\hline \multicolumn{2}{|l|}{ Hexactins } \\
\hline Diameter & $320-\underline{801}-1325(7)$ \\
\hline \multicolumn{2}{|l|}{ Pentactins } \\
\hline Length & - \\
\hline Diameter & - \\
\hline \multicolumn{2}{|l|}{ Macrouncinates } \\
\hline Length & $2125-\underline{4185}-7000(5)$ \\
\hline \multicolumn{2}{|l|}{ Microuncinates } \\
\hline Length & $33-\underline{41}-55(30)$ \\
\hline \multicolumn{2}{|l|}{ Pinular hexactins } \\
\hline Length & $500-748-925(11)$ \\
\hline Diameter & $290-401-485(11)$ \\
\hline \multicolumn{2}{|l|}{ Macramphidiscs } \\
\hline Length & $144-\underline{180}-210(13)$ \\
\hline Umbel length & $40-\underline{70}-88(13)$ \\
\hline Umbel width & $32-\underline{60}-80(13)$ \\
\hline \multicolumn{2}{|l|}{ Mesamphidiscs } \\
\hline Length & $93-\underline{122}-150(30)$ \\
\hline Umbel length & $15-\underline{30}-45(30)$ \\
\hline Umbel width & $16-\underline{27}-35(30)$ \\
\hline \multicolumn{2}{|l|}{ Micramphidiscs } \\
\hline Length & $47-\underline{62}-95(30)$ \\
\hline Umbel length & $5-\underline{11}-15(30)$ \\
\hline Umbel width & $8-\underline{12}-18(30)$ \\
\hline \multicolumn{2}{|l|}{ Microhexactins } \\
\hline Diameter & $95-\underline{158}-230(30)$ \\
\hline \multicolumn{2}{|l|}{ Micropentactins } \\
\hline Length & $45-\underline{96}-145(30)$ \\
\hline Diameter & $75-\underline{168}-240(30)$ \\
\hline \multicolumn{2}{|c|}{ Gastral pinular pentactins } \\
\hline Length & $150-\underline{164}-174(5)$ \\
\hline Basis diameter & $125-\underline{150}-180(5)$ \\
\hline \multicolumn{2}{|c|}{ Dermal pinular pentactins } \\
\hline Length & $205-\underline{252}-390(30)$ \\
\hline Basis diameter & $105-157-220(30)$ \\
\hline
\end{tabular}

